\title{
Outcomes for Patients with Glioblastoma Multiforme Treated with Chemoradiation Alone or Chemoradiation Followed by Adjuvant Chemotherapy
}

\author{
Magda Mostafa ${ }^{1}$, Ehab Saad ${ }^{1}$, Eiman Abdel-Hady ${ }^{1}$, Amr Amin ${ }^{2}$, Ashraf Monir ${ }^{1}$, Mostafa El- \\ Haddad.
}

${ }^{1}$ Department of Clinical Oncology, Kasr El-Aini Faculty of Medicine-Cairo University

${ }^{2}$ Department of Nuclear Medicine, Kasr El-Aini Faculty of Medicine-Cairo University

\begin{abstract}
Purpose: The aim of this work is to study the efficacy and toxicity of adjuvant temozolamide after concurrent temozolamide and radiatotherapy versus concurrent chemoradiation alone in patients with glioblastoma multiforme (GBM).

Patients and Methods: Forty patients with GBM were enrolled in our study at Kasr El-Ainy Center of Clinical Oncology and Nuclear Medicine. Nineteen patients were treated by concurrent temozolamide with radiotherapy followed by adjuvant temozolamide for 6 months. Another twenty one patients were treated by concurrent temozolamide with radiotherapy without 6 months of adjuvant temozolamide. A comparison of efficacy and toxicity was done between the two treatment arms.

Results: there was no significant difference $(p=0.81)$ in the median PFS between patients treated with radiation therapy and concomitant temozolamide followed by adjuvant temozolamide and patients treated with radiotherapy and concomitant temozolamide followed by observation. The median overall survival was also not statistically different between both schedules $(p=0.94)$. All patients receiving adjuvant temozolamide developed toxicity of different grades during adjuvant temozolamide treatment period. Common adverse events were acute grade 1 or 2 toxicities. Alopecia was reported in 12 out of 19 (63.2\%) patients followed by nausea (52.6\%) and fatigue (47.4\%). Only 4 (21\%) out of 19 patients experienced grade 3 toxicities. Two patients experienced grade 3 alopecia, one patient experienced grade 3 headache and one patient experienced grade 3 nausea.

Conclusion: Temozolamide in combination with radiation therapy followed by observation exhibit comparable efficacy in the term of progression free survival and overall survival in comparison to the standard arm, with less toxicity.
\end{abstract}

Key words: Concurrent temozolomide; adjuvant temozolomide; glioblastoma multiforme

Corresponding Author: Mostafa El-Haddad, MD., FRCR.

E-mail: mostafa_haddad@yahoo.co.uk

\section{INTRODUCTION}

Malignant gliomas are divided into anaplastic gliomas (anaplastic astrocytoma, anaplastic oligodendroglioma, and anaplastic oligoastrocytoma) and glioblastoma multiforme (GBM) based upon their histopathologic features ${ }^{1}$. GBM is best managed with a combined modality approach, incorporating maximal surgical resection, adjuvant postoperative radiation therapy and adjuvant chemotherapy ${ }^{2}$. The benefit of using temozolomide concurrently with radiation followed by adjuvant temozolomide compared with radiation alone was demonstrated in a phase III trial of 573 patients who underwent surgery for GBM. Temozolomide improved overall survival at two years (10 vs. $26 \%$, respectively $)^{3}$. The survival advantage in the European Organisation for Research and Treatment of Cancer (EORTC) trial may be attributed either to the temozolomide in the concurrent or to the adjuvant temozolomide or to the combined effect and there was no third arm to compare the effect of the concurrent versus the adjuvant treatment. The aim of our study was to compare the efficacy in terms of progression free survival (PFS) and overall survival (OS) and toxicity of temozolamide when given as adjuvant treatment for 6 months after its concurrent treatment with radiotherapy versus temozolamide concurrently with radiotherapy alone in patients with GBM.

\section{MATERIALS AND METHODS}

The study design was approved by our institutional scientific and ethical committees. A written consent was taken from all patients before their recruitment in the study.

\section{Pretreatment Evaluation}

The pretreatment evaluation included medical history, physical examination, laboratory studies: complete blood picture, basic chemistry, including serum calcium and liver function tests. Post-operative magnetic 
resonance imaging (MRI) of the brain as a baseline evaluation (4 weeks after surgery) and Technetium99m (V) dimercaptosuccinic acid (Tc-99m [V] DMSA) brain single photon emission computerized tomography (SPECT) as baseline evaluation (usually 4 weeks after surgery). SPECT images of the brain were obtained at $2-3 \mathrm{~h}$ after intravenous administration of $555-740 \mathrm{MBq}$ Tc-99m (V) DMSA using a dual head gamma camera (Philips Vertex-plus) fitted with a low energy high resolution collimator.

Extent of resection was based on the surgeons' judgement and postoperative brain MRI:

- Complete resection was defined as absence of contrastenhancing tumor on postoperative MRI.

- Residual tumor was defined as contrast enhancement with a volume more than $0.175 \mathrm{~cm} 3$ in the T1 image on postoperative MRI.

\section{Study design}

This is a prospective randomized pilot study including a total of 40 patients, 19 patients (arm A) received wide field radiotherapy technique concomitantly with temozolamide, $75 \mathrm{mg} / \mathrm{m}^{2}$ seven days per week for 42 days this was followed 4-weeks later by to six cycles of adjuvant oral temozolomide $150200-\mathrm{mg} / \mathrm{m}^{2}$ for 5 days every 28 days. The second group (Arm B) received same treatment without adjuvant temozolamide. Total radiation dose was 60 Gy / 30 fractions / 6 weeks with the gross tumor volume (GTV) included all MRI detected enhanced lesion. Clinical target volume (CTV) included 23- $\mathrm{cm}$ margin around the gross disease and the postoperative cavity.

\section{Post treatment follow-up}

Follow up MRI brain and SPECT scan were done 1 month after the end of the concurrent chemo-radiation and every 3 months thereafter. Toxicity was reported according to the National Cancer Institute common toxicity criteria.

\section{Statistical methods}

Comparison of toxicity was done using Fisher's exact test. The OS was calculated from the date of diagnosis till date of death. PFS was calculated from the date of diagnosis till the date of documented disease progression. Both OS and PFS were computed by the Kaplan-Meier method and compared by the log-rank test and the Cox proportional hazards model. $P$ values less than 0.05 was considered statistically significant. The multivariate Cox model was used to study variation in the OS and PFS according to major baseline characteristics (age, sex, stage, histology and treatment). Statistical analyses were conducted using SPSS software, version 13.0 (SPSS, Inc, Chicago, IL, USA).

\section{RESULTS}

Between January 2012 and January 2014, 40 patients with GBM were enrolled in our study at Kasr El-Ainy Center of Clinical Oncology and Nuclear Medicine (NEMROCK). Patients and tumors characteristics are shown in Tables 1 and 2 respectively.

The estimated median PFS for the whole group of patients was 10.4 months (95\%CI: 9.511.3-) (Figure 1). There was no statistically significant difference in median PFS for both groups [10.4 months (95\% CI: 9.811-) for group A and 10.37 months (95\%CI: 7.713.1-) ( $p$ value 0.81 ) for group B] (Figure 2). In univariate analysis, PFS was found to be longer in patients: younger than 50 years (11.3 versus 7.5 months) ( $p=0.001)$, with tumor residual less than $20 \mathrm{cc}(15.8$ versus 9.3 months $)(p=0.02)$, with surgical resection than biopsy (11.1 versus 8.4 months) $(p<0.001)$, patients with negative pretreatment DMSA (11.3 versus 8.6 months) ( $p=0.003)$, and among patients with negative DMSA scan at 6 months (15.8 versus 10.4 months) $(p=0.002)$. However, these variables were not significant on multivariate analysis. The results of the univariate and multivariate analysis are summarized in Table 3 .

The estimated median OS for the whole group of patients was 12.8 months (95\%CI: 10.914.7-) (Figure 3). The estimated median OS for group A was 13.2 months (95\%CI: 10.915.5-) and for group B was 12.8 months (95\%CI: 8.417.2-). There was no statistically significant difference between the two treatment arms ( $\mathrm{P}$ value 0.94 ) (Figure 4). In univariate analysis, overall survival was found to be better for in patients younger than 50 years (14.4 versus 8.9 months) $(p$ value $<0.001)$, with tumor residual less than $20 \mathrm{cc}$ (14.4 versus 10.7 months) ( $p$ value $0.008)$, with surgical resection than biopsy (14.4 versus 8.8 months) ( $p$ value $<0.001$ ), patients with negative pretreatment DMSA (median OS not reached versus 10.4 months) ( $p$ value $<0.001$ ), and among patients with KPS >80 (16.7 versus 11.3 months) ( $p$ value 0.003$)$. In multivariate analysis, age, surgical extent, and the pretreatment DMSA scan were independent predictors of OS. The results of the univariate and multivariate analysis are summarized in Table 4.

All patients completed the specified course of radiation therapy. The median duration of treatment in the whole group of patients was 46 days (range: 4250days). There was no significant difference between the mean duration of treatment in both arms $(p=0.5)$. The treatment was interrupted in one patient for one week due to scalp infection which was managed successfully by local and systemic antibiotics. Total dose of concurrent temozolamide was given to all patients without need for dose modification. The commonest reported toxicities 
included headache in $22(55 \%)$ patients followed by alopecia (47.5\%) and malaise (40\%), with no significant differences between the treatment arms. In Arm A, 2 patients experienced grade 3 alopecia and one patient experienced grade 3 headache. In Arm B, one patient experienced grade 3 headache and one patient developed grade 3 malaise. The main adverse events in patients randomized across 2 groups are summarized in Table 5 .

The commonest temozolamide related toxicity included: nausea $(52.6 \%)$ and fatigue $(47.4 \%)$. Toxicities with adjuvant temozolamide treatment are illustrated in Table 6.

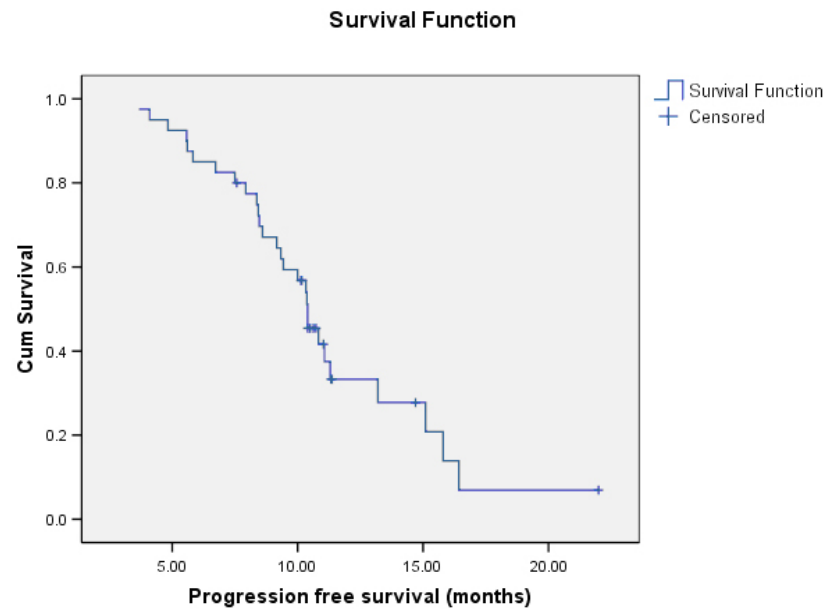

Figure. 1: Progression free survival of the whole group of patients.

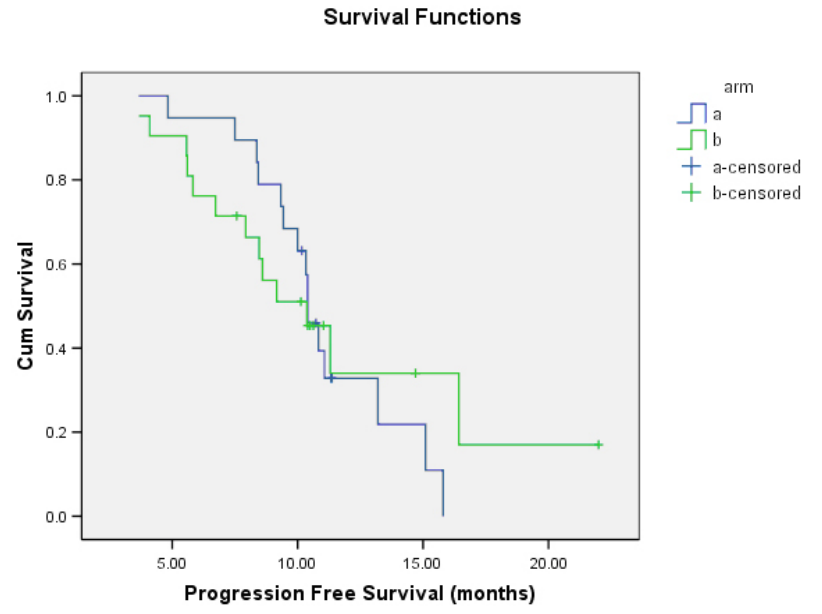

Figure. 2: Progression free survival according the two treatment arms ( $p$ value 0.81 )

${ }^{*}$ Arm A: Patients who received concurrent chemoradiation followed by adjuvant treatment

*Arm B: Patients who received concurrent chemoradiation without adjuvant treatment 


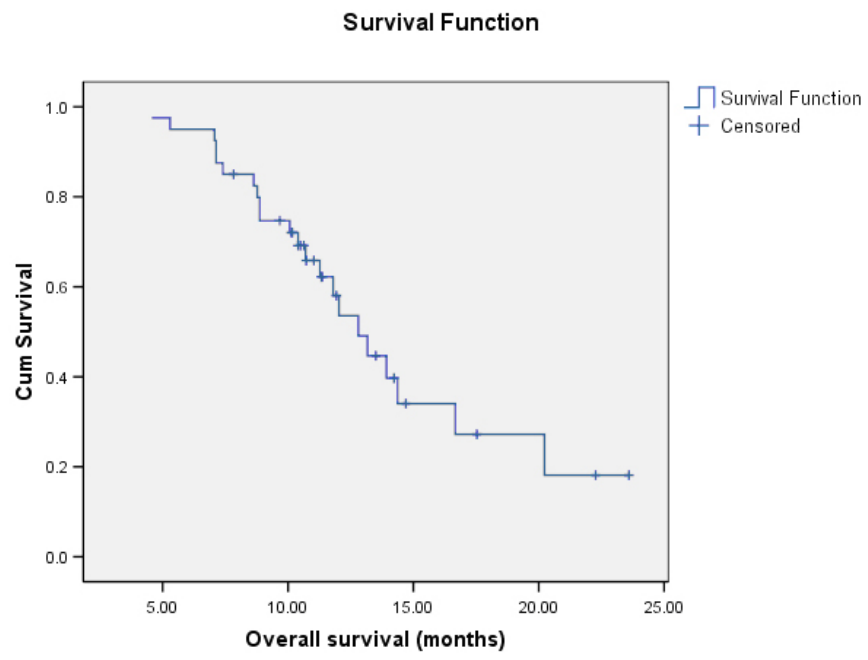

Figure. 3: Overall survival of the whole group of patients.

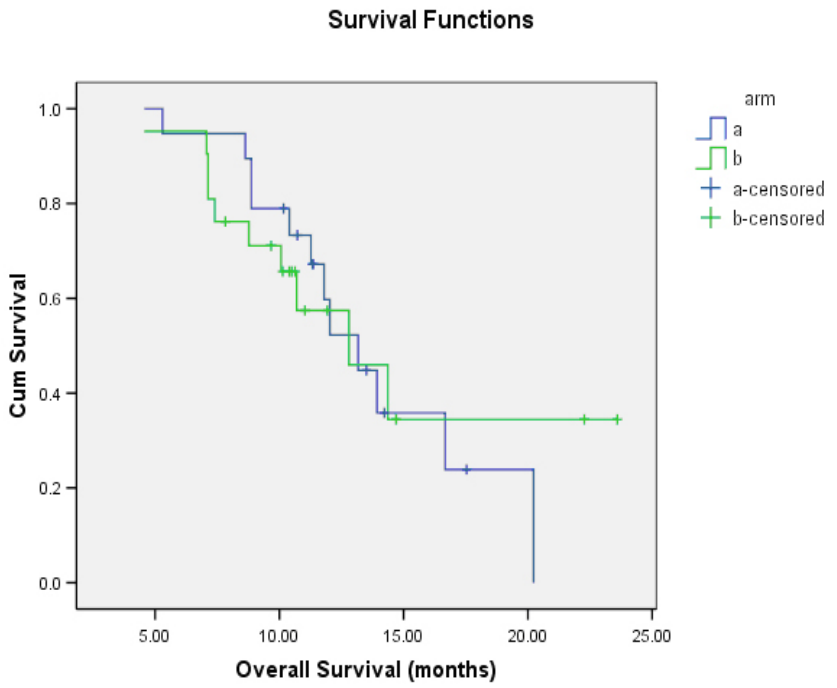

Figure. 4: Overall survival according to treatment arms ( $P$ value 0.94$)$.

*Arm A: Patients who received concurrent chemoradiation followed by adjuvant treatment.

*Arm B: Patients who received concurrent chemoradiation without adjuvant treatment. 
Table 1: Patient characteristics.

\begin{tabular}{|c|c|c|c|}
\hline & $\operatorname{Arm} A(n=19), n(\%)$ & $\operatorname{Arm} B(n=21), n(\%)$ & $P$ value \\
\hline \multicolumn{4}{|l|}{ Age } \\
\hline Mean $\pm \mathrm{SD}$ & $43.5 \pm 11.9$ & $48.6 \pm 12.2$ & 0.187 \\
\hline$<50$ & $14(73.7)$ & $12(57.1)$ & 0.273 \\
\hline$\geq 50$ & $5(26.3)$ & $9(42.9)$ & \\
\hline \multicolumn{4}{|l|}{ Sex } \\
\hline Male & $10(53 \%)$ & $11(52 \%)$ & 0.99 \\
\hline Female & $9(47 \%)$ & $10(48 \%)$ & \\
\hline \multicolumn{4}{|l|}{ ECOG performance status } \\
\hline 70 & $5(26.3)$ & $6(28.6)$ & 0.825 \\
\hline 80 & $9(47.4)$ & $8(38.1)$ & \\
\hline 90 & $5(26.3)$ & $7(33.3)$ & \\
\hline \multicolumn{4}{|l|}{ Symptoms } \\
\hline Headache & $15(78.9)$ & $16(76.2)$ & 0.835 \\
\hline Vomiting & $4(21.1)$ & $4(19)$ & 0.874 \\
\hline Blurred vision & $4(21.1)$ & $5(23.8)$ & 0.835 \\
\hline Weakness & $8(42.1)$ & $9(42.9)$ & 0.962 \\
\hline Urine incontinence & $1(5.3)$ & $2(9.5)$ & 0.609 \\
\hline Diminished vision & $1(5.3)$ & $1(4.8)$ & 0.942 \\
\hline Slurred speech & $1(5.3)$ & $3(14.3)$ & 0.342 \\
\hline Decreased consciousness & $3(15.8)$ & 0 & 0.58 \\
\hline Convulsions & $5(26.3)$ & $7(33.3)$ & 0.629 \\
\hline \multicolumn{4}{|l|}{ Neurological signs } \\
\hline Any & $9(47.4)$ & $14(66.7)$ & 0.218 \\
\hline Hemiparesis & $8(42.1)$ & $9(42.9)$ & 0.962 \\
\hline Papilledema & $1(5.3)$ & $4(19)$ & 0.188 \\
\hline \multicolumn{4}{|l|}{ Surgical procedure } \\
\hline Biopsy & $6(31.6)$ & $5(23.8)$ & 0.787 \\
\hline Complete resection & $1(5.3)$ & $2(9.5)$ & \\
\hline Partial resection & $12(63.2)$ & $14(66.7)$ & \\
\hline \multicolumn{4}{|l|}{ Pre-treatment DMSA ${ }^{*}$} \\
\hline Negative & $10(55.6)$ & $6(28.6)$ & 0.09 \\
\hline Positive & $8(44.4)$ & $15(71.4)$ & \\
\hline
\end{tabular}

Table 2: Tumor characteristics.

\begin{tabular}{|c|c|c|c|}
\hline & $\operatorname{Arm} A(n=19), n(\%)$ & $\operatorname{Arm} B(n=21), n(\%)$ & $P$ value \\
\hline \multicolumn{4}{|l|}{ Site } \\
\hline Frontal & $3(15.8)$ & $5(23.8)$ & 0.698 \\
\hline Parietal & $12(63.2)$ & $13(61.9)$ & 0.935 \\
\hline Occipital & $2(10.5)$ & $4(19)$ & 0.664 \\
\hline Temporal & $9(47.4)$ & $9(42.9)$ & 0.775 \\
\hline \multicolumn{4}{|l|}{ Side } \\
\hline Left & $9(47.4)$ & $9(42.9)$ & 0.775 \\
\hline Right & $10(52.6)$ & $12(57.1)$ & \\
\hline \multicolumn{4}{|c|}{ Pretreatment volume (cc) } \\
\hline Mean $( \pm \mathrm{SD})$ & $49.7( \pm 11.6)$ & $50.6( \pm 11)$ & 0.795 \\
\hline \multicolumn{4}{|l|}{ Biopsy volume (cc) } \\
\hline Mean $( \pm \mathrm{SD})$ & $23.8( \pm 10.8)$ & $21( \pm 28.9)$ & 0.212 \\
\hline \multicolumn{4}{|c|}{ Post-surgery volume (cc) } \\
\hline Mean $( \pm \mathrm{SD})$ & $25.8( \pm 17)$ & $21.7( \pm 15.2)$ & 0.422 \\
\hline
\end{tabular}


Kasr-El-Aini Journal Of Clinical Oncology And Nuclear Medicine

Table 3: Progression Free Survival according to different variables in univariate and multivariate analysis.

\begin{tabular}{|c|c|c|c|c|c|}
\hline \multirow{2}{*}{ Variable } & \multirow{2}{*}{ Group } & \multirow{2}{*}{ N. } & \multirow{2}{*}{ Median PFS in months (95\% CI) } & \multicolumn{2}{|c|}{ P value } \\
\hline & & & & Univariate & Multivariate * \\
\hline \multirow{2}{*}{ Age (years) } & $<50$ & 26 & $11.3(8.9-13.7)$ & 0.001 & 0.068 \\
\hline & $\geq 50$ & 14 & $7.5(5.3-9.7)$ & & \\
\hline \multirow{2}{*}{ Sex } & Female & 19 & $10(8.7-11.4)$ & 0.677 & na \\
\hline & Male & 21 & $10.8(9.9-11.7)$ & & \\
\hline \multirow{2}{*}{ Pre-surgery volume (cc) } & $<50$ & 17 & $11.3(9-13.6)$ & 0.526 & na \\
\hline & $\geq 50$ & 23 & $10.4(9-11.7)$ & & \\
\hline \multirow{2}{*}{ Surgery extent } & Biopsy & 11 & $8.4(5.6-11.2)$ & $<0.001$ & 0.149 \\
\hline & Resection & 29 & $11.1(9.9-12.2)$ & & \\
\hline \multirow{2}{*}{ Post-surgery residual volume (cc) } & $<20$ & 15 & $15.8(10.7-20.9)$ & 0.022 & 0.688 \\
\hline & $\geq 20$ & 25 & $9.3(8-10.6)$ & & \\
\hline \multirow{2}{*}{ KPS } & $\leq 80$ & 28 & $9.3(7.6-11.1)$ & 0.001 & 0.083 \\
\hline & $>80$ & 12 & $15.8(7.4-24.2)$ & & \\
\hline \multirow{2}{*}{ Total CCRT treatment time } & $<7$ weeks & 35 & $10.4(9.9-10.9)$ & 0.766 & na \\
\hline & $\geq 7$ weeks & 5 & $15.1(-)$ & & \\
\hline \multirow{2}{*}{$\begin{array}{l}\text { Pre-treatment DMSA } \\
(39 \text { pts.) }\end{array}$} & Negative & 16 & $11.3(6.2-16.4)$ & 0.003 & 0.454 \\
\hline & Positive & 23 & $8.6(7.5-9.7)$ & & \\
\hline \multirow{2}{*}{$\begin{array}{l}\text { DMSA at } 6 \text { months } \\
\text { (32 pts.) }\end{array}$} & Negative & 11 & $15.8(9.2-22.4)$ & 0.002 & ------- \\
\hline & Positive & 21 & $10.4(8.4-12.3)$ & & \\
\hline \multirow{2}{*}{ Treatment arm } & $\mathrm{A}$ & 19 & $10.4(9.8-11)$ & 0.81 & na \\
\hline & $\mathrm{B}$ & 21 & $10.37(7.7-13.1)$ & & \\
\hline
\end{tabular}

"Excluding one patient for whom pre-DMSA was not done and did not include post DMSA.

Table 4: Overall Survival according to different variables in univariate and multivariate analyses.

\begin{tabular}{|c|c|c|c|c|c|}
\hline \multirow{2}{*}{ Variable } & \multirow{2}{*}{ Group } & \multirow{2}{*}{ N. } & \multirow{2}{*}{ Median OS in months $(95 \% \mathrm{CI})$} & \multicolumn{2}{|c|}{ P value } \\
\hline & & & & Univariate & Multivariate $^{*}$ \\
\hline \multirow{2}{*}{ Age (years) } & $<50$ & 26 & $14.4(10.8-17.9)$ & $<0.001$ & 0.029 \\
\hline & $\geq 50$ & 14 & $8.9(6.7-11.1)$ & & \\
\hline \multirow{2}{*}{ Sex } & Female & 19 & $12(9.7-14.3)$ & 0.725 & na \\
\hline & Male & 21 & $13.9(10.4-17.5)$ & & \\
\hline \multirow{2}{*}{ Pre-surgery volume (cc) } & $<50$ & 17 & $13.9(11.4-16.5)$ & 0.27 & na \\
\hline & $\geq 50$ & 23 & $11.8(10.5-13.1)$ & & \\
\hline \multirow{2}{*}{ Surgery extent } & Biopsy & 11 & $8.8(7.6-10)$ & $<0.001$ & 0.014 \\
\hline & Resection & 29 & $14.4(10.8-17.9)$ & & \\
\hline \multirow{2}{*}{ Post-surgery residual volume (cc) } & $<20$ & 15 & $14.4(-)$ & 0.008 & 0.978 \\
\hline & $\geq 20$ & 25 & $10.7(7.4-14)$ & & \\
\hline \multirow{2}{*}{ KPS } & $\leq 80$ & 28 & $11.3(8.9-13.6)$ & 0.003 & 0.757 \\
\hline & $>80$ & 12 & $16.7(-)$ & & \\
\hline \multirow{2}{*}{ Total CCRT treatment time } & $<7$ weeks & 35 & $12.8(11-14.6)$ & 0.873 & na \\
\hline & $\geq 7$ weeks & 5 & $20.2(-)$ & & \\
\hline \multirow{2}{*}{$\begin{array}{l}\text { Pre-treatment DMSA } \\
\text { (39 pts.) }\end{array}$} & Negative & 16 & Not reached & $<0.001$ & 0.033 \\
\hline & Positive & 23 & $10.4(8-12.8)$ & & \\
\hline \multirow{2}{*}{$\begin{array}{l}\text { DMSA at } 6 \text { months } \\
\text { (32 pts.) }\end{array}$} & Negative & 11 & Not reached & 0.062 & ----- \\
\hline & Positive & 21 & $13.9(9.8-18.1)$ & & \\
\hline \multirow{2}{*}{ Treatment arm } & $\mathrm{A}$ & 19 & $13.2(10.9-15.5)$ & 0.941 & na \\
\hline & $\mathrm{B}$ & 21 & $12.8(8.4-17.2)$ & & \\
\hline
\end{tabular}

"Excluding one patient for whom pre-DMSA was not done and did not include post DMSA.

Table 5: Toxicity during concurrent chemoradiation.

\begin{tabular}{|c|c|c|c|c|c|c|c|c|}
\hline & \multirow{3}{*}{$\begin{array}{l}\text { Any grade } \\
\text { Total } \\
\text { n (\%) } \\
\end{array}$} & \multirow[t]{2}{*}{ Grade 3, 4} & \multirow{2}{*}{$\begin{array}{l}\text { Any grade } \\
\text { Arm A } \\
\end{array}$} & \multicolumn{5}{|c|}{ Grade 3, 4} \\
\hline & & & & Arm B & \multirow{2}{*}{ P value } & Arm A & Arm B & \multirow{2}{*}{ P value } \\
\hline & & & n (\%) & & & n (\%) & & \\
\hline Alopecia & $19(47.5)$ & $2(5)$ & $10(52.6)$ & $9(42.9)$ & 0.536 & $2(10.5)$ & 0 & 0.219 \\
\hline Convulsions & $8(20)$ & 0 & $4(21.1)$ & $4(19)$ & 1 & 0 & 0 & --- \\
\hline Decreased conscious level & $2(5)$ & 0 & $1(5.3)$ & $1(4.8)$ & 1 & 0 & 0 & --- \\
\hline Headache & $22(55)$ & $2(50)$ & $10(52.6)$ & $12(57.1)$ & 0.775 & $1(5.3)$ & $1(4.8)$ & 1 \\
\hline Malaise & $16(40)$ & $1(2.5)$ & $6(31.6)$ & $10(47.6)$ & 0.301 & 0 & $1(4.8)$ & 1 \\
\hline Nausea & $14(35)$ & 0 & $8(42.1)$ & $6(28.6)$ & 0.37 & 0 & 0 & --- \\
\hline Vomiting & $8(20)$ & 0 & $4(21.1)$ & $4(19)$ & 1 & 0 & 0 & --- \\
\hline Tingling & $4(10)$ & 0 & $1(5.3)$ & $3(14.3)$ & 0.607 & 0 & 0 & --- \\
\hline
\end{tabular}


Kasr-El-Aini Journal Of Clinical Oncology And Nuclear Medicine

Vol. 10 | No. 3-4 $2014 \quad$ Mostafa El-Haddad et al

Table 6: Toxicity during adjuvant temozolamide treatment.

\begin{tabular}{lcc}
\hline & Any grade & Grade 3,4 \\
\cline { 2 - 3 } & \multicolumn{2}{c}{ n (\%) } \\
\hline Alopecia & $12(63.2)$ & $2(10.5)$ \\
\hline Anorexia & $5(26.3)$ & 0 \\
\hline Fatigue & $9(47.4)$ & 0 \\
\hline Headache & $6(31.6)$ & $1(5.3)$ \\
\hline Nausea & $10(52.6)$ & $1(5.3)$ \\
\hline Neutropenia & $2(10.5)$ & 0 \\
\hline Thrombocytopenia & $1(5.3)$ & 0 \\
\hline Vomiting & $6(31.6)$ & 0 \\
\hline
\end{tabular}

\section{DISCUSSION}

For many years, a lot of efforts and attempts were done to improve the prognosis of patients with $\mathrm{GBM}^{4}$. In the late1990's, temozolamide seemed promising for the treatment of recurrent $\mathrm{GBM}^{5,6}$. A pilot phase II study showed that concomitant temozolamide with conventionally fractionated radiotherapy, followed by six cycles as adjuvant therapy was feasible. ${ }^{7}$ The EORTCNCIC trial published in 2009 showed that the benefits of concurrent-adjuvant temozolamide with radiotherapy were maintained throughout the 5 years of follow up, with a 5 -year survival rate of $9.8 \%$ versus $1.9 \%$ for those treated with radiation therapy alone. Median OS rates for radiotherapy with concurrent temozolamide followed by adjuvant temozolamide versus radiotherapy alone were 14.6 months and 12.1 months respectively. PFS for patients receiving radiotherapy/temozolamide compared with radiotherapy alone were $11.2 \%$ and $1.8 \%$ at 2 years, $4.1 \%$ and $1.3 \%$ at 5 years respectively ${ }^{3}$.

Although that study changed the standard of care, there is a question still searching for an answer, which is the contribution of each of the concomitant and the adjuvant temozolamide, which is now being investigated in the ongoing EORTC-Intergroup trial on anaplastic astrocytoma (CATNON trial), which started recruiting patients in November, $2011^{8}$. Our study asked the same question but the number of patients is small to give a final answer, however, it highlight that the survival for those receiving the concurrent temozolamide only may not be worse than those received adjuvant treatment in addition to the adjuvant treatment 13.2 months [ $(95 \%$ CI: 10.9 $15.5)]$ and 12.8 months [(95\%CI: 8.417.2-)] respectively. The estimated median OS for the whole group of patients was 12.8 months (95\%CI: 10.914.7-) which may be comparable to data released by EORTC-NCI trial of median survival (14.6 months). Survival in GBM do not show plateau and most of the patients progress and die of the disease and most of the patients will be treated by chemotherapy, so the question also may be the value of adjuvant chemotherapy versus salvage or early versus delayed treatment with chemotherapy. Hart et al evaluated the effect of temozolomide in a Cochrane metaanalysis and showed that it increases the OS and PFS with a hazard ratio (HR) of $0.60[(95 \% \quad \mathrm{CI}=0.46$ to $0.79, P$ value 0.0003$)]$ and $0.63[(95 \% \mathrm{CI}=0.43$ to $0.92, P$ value 0.02] respectively, when compared with radiotherapy alone, although these benefits only appeared to emerge when therapy is given in both concomitant and adjuvant phases of treatment with increased of haematological complications, fatigue and infections ${ }^{9}$. In our series patients tolerated treatment very well with only $3(15 \%)$ out of 19 patients in arm A and $2(9 \%)$ out of 21 patients of arm $\mathrm{B}$ experienced grade 3 toxicities.

Tc-99m (V) DMSA is a tumor-seeking radiotracer displaying affinity for gliomas. In our group of patients, pre-treatment DMSA scan was found to negative in 16 patients and was found to be an independent predictor of OS in multivariate analysis for the whole group of patients, ( $p$ value 0.03 ). This may be a surrogate for adequate surgery hence better survival; however it may have a value to stratify the patients into low risk or high risk. The main limitation of this work is the relatively small number of cases and short follow up time. Confirmation study is required to validate our findings.

\section{CONCLUSION}

Adjuvant chemotherapy following concurrent chemoradiation may not add to GBM patients' survival, however longer follow-up for our patients and larger number may be needed to confirm our results. DMSA scan may be of important diagnostic and prognostic value and may need further evaluation with bigger number of patients.

\section{REFERENCES}

1. Janzer RC. Neuropathology and molecular pathology of gliomas. Rev Med Suisse. 2009;5:1501-1504.

2. Laws ER, Parney IF, Huang W, et al. Survival following surgery and prognostic factors for recently diagnosed malignant glioma: data from the Glioma Outcomes Project. J Neurosurg. 2003;99:467-473.

3. Stupp R, Hegi ME, Mason WP, et al. Effects of radiotherapy with concomitant and adjuvant temozolomide versus radiotherapy alone on survival in glioblastoma in a randomised phase III study: 5-year analysis of the EORTC-NCIC trial. Lancet Oncol. 2009; 10:459-466.

4. Laperriere N, Zuraw L, Cairncross G, et al. Radiotherapy for newly diagnosed malignant glioma in adults: a systematic review. Radiother Oncol. 2002;64:259-273.

5. Newlands ES, Goldman S, Dewitte O, et al. Temozolomide: a review of its discovery, chemical properties, pre-clinical development and clinical trials. Cancer Treat Rev. 1997;23:35-61. 
6. Brock CS, Beaney RP, Thomas DG, et al. Phase I trial of temozolomide using an extended continuous oral schedule. Cancer Res. 1998;58:4363-4367.

7. Stupp R, Hegi ME, Mason WP, et al. Promising survival for patients with newly diagnosed glioblastoma multiforme treated with concomitant radiation plus temozolomide followed by adjuvant temozolomide. J Clin Oncol. 2002;20:1375-1382.
8. Wick $\mathrm{W}$ and Weller $\mathrm{M}$. Anaplastic glioma. Neuropathology, molecular diagnostics and current study concepts. Nervenarzt. 2010;81:928-30, 932-5.

9. Hart MG, Kofler J, Kelly LM, et al. Temozolomide for high grade glioma. Cochrane Database Syst Rev. 2013;4:CD007415. 